\title{
Aggregation-induced delayed fluorescence from phenothiazine-containing donor-acceptor molecules for high-efficiency non-doped organic light-emitting diodes
}

\begin{abstract}
Naoya Aizawa ${ }^{1}$, Chao-Jen Tsou ${ }^{1,2}$, In Seob Park ${ }^{1,3}$ and Takuma Yasuda ${ }^{1,2,3}$
Highly luminescent donor-acceptor molecules based on a phenothiazine donor unit coupled with a xanthone or benzophenone acceptor unit were developed for use in organic light-emitting diodes (OLEDs). While both molecules are almost non-luminescent in pure tetrahydrofuran (THF) solution, a strong yellow delayed fluorescence was observed upon their aggregation in THF/water mixtures or in neat films. This result demonstrates the unique aggregation-induced delayed fluorescence (AIDF) characteristics of these molecules. OLEDs using these AIDF materials as a non-doped emission layer achieved high external electroluminescence quantum efficiencies of up to $11 \%$, which exceeds the theoretical maximum for conventional fluorescent OLEDs.
\end{abstract}

Polymer Journal (2017) 49, 197-202; doi:10.1038/pj.2016.82; published online 21 September 2016

\section{INTRODUCTION}

Solid-state organic fluorophores have been actively explored for various optoelectronic applications, in particular for organic lightemitting diodes (OLEDs). ${ }^{1}$ Although most organic fluorophores are highly luminescent in dilute solutions, their luminescence is generally weakened or quenched in the solid state via bimolecular recombination and energy transfer to non-luminescent impurities, which results in a substantial energy loss in the OLEDs. ${ }^{2-5}$ This problem is typically circumvented by dispersing the fluorophores in wide bandgap host materials via precise co-evaporation processes; however, this approach requires complex manufacturing procedures. Thus, highly luminescent solid-state fluorophores are highly desirable for simplifying the device structure and the fabrication process.

In 2001, Tang and co-workers,7 identified materials that exhibit aggregation-induced emission (AIE). In contrast to conventional fluorophores, these AIE-active fluorophores are almost nonluminescent in dilute solutions, yet they become highly luminescent upon molecular aggregation. This AIE phenomenon can be explained by the restriction of non-radiative vibrational relaxation processes in the aggregated solid state. Representative examples of AIE-active fluorophores include siloles, ${ }^{6-9}$ cyanostilbenes, ${ }^{10-12}$ $o$-carborane derivatives ${ }^{13-17}$ and tetraphenylethenes ${ }^{18-23}$. Some of these fluorophores have proved useful as non-doped emission layers in fluorescent OLEDs. ${ }^{19-23}$ However, these OLEDs can only utilize singlet excitons for electroluminescence (EL) and the resulting internal EL quantum efficiency $\left(\eta_{\text {int }}\right)$ is limited to $25 \%$ because of the inherent spin-statistical limitation of electrical excitation, which generates radiative singlet excitons and non-radiative triplet excitons in a $1: 3$ ratio. $^{24}$

Recent research efforts in our group ${ }^{25}$ and others ${ }^{26-30}$ have focused on exploring AIE-active materials that simultaneously exhibit thermally activated delayed florescence ${ }^{31-39}$ to harvest triplet excitons for EL via efficient reverse intersystem crossing. To expand the library of such unique fluorophores that display aggregation-induced delayed fluorescence (AIDF), we report on two yellow AIDF materials, PTZ-XT and PTZ-BP (Figure 1) that consist of an electron-donating phenothiazine unit coupled with an electron-accepting xanthone or benzophenone unit. We demonstrate that these new AIDF materials can achieve high external EL quantum efficiencies $\left(\eta_{\text {ext }}\right)$ of up to $11 \%$ in non-doped OLEDs without any host materials.

\section{EXPERIMENTAL PROCEDURE}

Synthesis and characterization

Commercially available reagents and solvents were used without further purification unless otherwise noted. All of the reactions were performed under a nitrogen atmosphere in dry solvents using standard Schlenk techniques. High-performance liquid chromatography was performed using TSKgel ODS-120A (Tosoh, Tokyo, Japan). Nuclear magnetic resonance (NMR) spectra were recorded using an Avance III 400 spectrometer (Bruker Daltonics, Leipzig, Germany). Chemical shifts of ${ }^{1} \mathrm{H}$ and ${ }^{13} \mathrm{C}$ NMR signals were determined relative to the signals of tetramethylsilane $(\delta=0.00), \mathrm{CDCl}_{3}(\delta=77.0)$ and DMSO- $d_{6}(\delta=39.5)$, which were used as internal standards. Matrix-assisted laser desorption ionization time-of-flight (MALDI-TOF) mass spectra were collected using an Autoflex III spectrometer (Bruker Daltonics) with dithranol

\footnotetext{
${ }^{1}$ INAMORI Frontier Research Center (IFRC), Kyushu University, Fukuoka, Japan; ${ }^{2}$ Department of Automotive Science, Graduate School of Integrated Frontier Sciences, Kyushu University, Fukuoka, Japan and ${ }^{3}$ Department of Applied Chemistry, Graduate School of Engineering, Kyushu University, Fukuoka, Japan Correspondence: Dr N Aizawa or Professor T Yasuda, INAMORI Frontier Research Center (IFRC), Kyushu University, 744 Motooka, Nishi-ku, Fukuoka 819-0395, Japan. E-mail: aizawa@ifrc.kyushu-u.ac.jp or yasuda@ifrc.kyushu-u.ac.jp 
<smiles>O=c1c2ccccc2oc2cc(N3c4ccccc4Sc4ccccc43)ccc12</smiles>

PTZ-XT<smiles>O=C(c1ccccc1)c1ccc(N2c3ccccc3Sc3ccccc32)cc1</smiles>

PTZ-BP
Figure 1 Molecular structures of PTZ-XT and PTZ-BP.

as a matrix. Elemental analysis was carried out using a MT-5 CHN Corder (Yanaco, Tokyo, Japan).

\section{Synthesis of PTZ-XT}

A mixture of $10 \mathrm{H}$-phenothiazine $(1.99 \mathrm{~g}, 10.0 \mathrm{mmol})$, 3-bromoxanthone $(2.49 \mathrm{~g}, \quad 9.10 \mathrm{mmol}), \quad t$-BuONa $(1.92 \mathrm{~g}, 20.0 \mathrm{mmol}), \quad \mathrm{Pd}(\mathrm{OAc})_{2} \quad(0.05 \mathrm{~g}$, $0.22 \mathrm{mmol})$ and $\mathrm{P}(t-\mathrm{Bu})_{3} \cdot \mathrm{HBF}_{4}(0.32 \mathrm{~g}, 1.10 \mathrm{mmol})$ was stirred for $15 \mathrm{~h}$ at $110^{\circ} \mathrm{C}$. After cooling to room temperature, the reaction mixture was poured into water and then extracted with toluene. The combined organic layers were washed with water and dried over anhydrous $\mathrm{Na}_{2} \mathrm{SO}_{4}$. After filtration and evaporation, the product was purified by silica-gel column chromatography (eluent: dichloromethane) to afford PTZ-XT as a yellow solid (3.20 g, 89\%). This compound was further purified by temperature-gradient sublimation under vacuum. ${ }^{1} \mathrm{H}$ NMR $\left(400 \mathrm{MHz}, \mathrm{DMSO}-d_{6}\right): \delta 8.14(\mathrm{dd}, J=7.9,1.6 \mathrm{~Hz}$, $1 \mathrm{H}), 8.05(\mathrm{~d}, J=8.8 \mathrm{~Hz}, 1 \mathrm{H}), 7.80(\mathrm{td}, J=7.8,1.8 \mathrm{~Hz}, 1 \mathrm{H}), 7.65-7.55$ $(\mathrm{m}, 5 \mathrm{H}), 7.50(\mathrm{td}, J=7.7,1.5 \mathrm{~Hz}, 2 \mathrm{H}), 7.43(\mathrm{td}, J=7.5,1.0 \mathrm{~Hz}, 1 \mathrm{H}), 7.37$ (td, $J=7.6,1.3 \mathrm{~Hz}, 2 \mathrm{H}), 7.01(\mathrm{dd}, J=8.9,2.4 \mathrm{~Hz}, 1 \mathrm{H}), 6.92(\mathrm{~d}, J=2.5 \mathrm{~Hz}, 1 \mathrm{H})$. ${ }^{13} \mathrm{C}$ NMR $\left(125 \mathrm{MHz}, \mathrm{CDCl}_{3}\right): \delta 175.97,157.76,156.20,151.39,140.90,134.18$, $134,04,129.01,128.13,127.46,126.69,126.62,126.46,123.72,122.08,117.55$, 115.85, 112.65, 102.19. MS (MALDI-TOF): $\mathrm{m} / \mathrm{z}[\mathrm{M}]^{+}$calcd 393.08; found, 392.72. Anal. Calcd (\%) for $\mathrm{C}_{25} \mathrm{H}_{15} \mathrm{NO}_{2} \mathrm{~S}$ : C, $76.32 ; \mathrm{H}, 3.84 ; \mathrm{N}, 3.56$; found: $\mathrm{C}$, $76.38 ; \mathrm{H}, 3.83 ; \mathrm{N}, 3.60$.

\section{Synthesis of PTZ-BP}

A mixture of $10 \mathrm{H}$-phenothiazine $(1.99 \mathrm{~g}, 10.0 \mathrm{mmol}), 4$-bromobenzophenone $(2.38 \mathrm{~g}, \quad 9.10 \mathrm{mmol}), \quad t$-BuONa $(1.92 \mathrm{~g}, \quad 20.0 \mathrm{mmol}), \quad \mathrm{Pd}(\mathrm{OAc})_{2} \quad(0.05 \mathrm{~g}$, $0.22 \mathrm{mmol})$ and $\mathrm{P}(t-\mathrm{Bu})_{3} \cdot \mathrm{HBF}_{4}(0.32 \mathrm{~g}, 1.10 \mathrm{mmol})$ was stirred for $24 \mathrm{~h}$ at $110^{\circ} \mathrm{C}$. After cooling to room temperature, the reaction mixture was poured into water and then extracted with toluene. The combined organic layers were washed with water and dried over anhydrous $\mathrm{Na}_{2} \mathrm{SO}_{4}$. After filtration and evaporation, the product was purified by silica-gel column chromatography (eluent: dichloromethane $/ n$-hexane $=1: 2, \quad \mathrm{v} / \mathrm{v}$ ) to obtain PTZ-BP as a yellow solid $(2.52 \mathrm{~g}, 73 \%)$. This compound was further purified by temperature-gradient sublimation under vacuum. ${ }^{1} \mathrm{H}$ NMR $\left(400 \mathrm{MHz}, \mathrm{CDCl}_{3}\right)$ : $\delta 7.84(\mathrm{~d}, J=9.0,2.4 \mathrm{~Hz}, 2 \mathrm{H}), 7.79(\mathrm{dd}, J=8.4,1.4 \mathrm{~Hz}, 2 \mathrm{H}), 7.57(\mathrm{tt}, J=7.4$, $1.6 \mathrm{~Hz}, 1 \mathrm{H}), 7.48(\mathrm{td}, J=7.3,1.5 \mathrm{~Hz}, 2 \mathrm{H}), 7.32(\mathrm{dd}, J=7.5,1.3 \mathrm{~Hz}, 2 \mathrm{H})$, $7.24(\mathrm{~d}, J=9.0,2.3 \mathrm{~Hz}, 2 \mathrm{H}), 7.18(\mathrm{td}, J=7.3,1.5 \mathrm{~Hz}, 2 \mathrm{H}), 7.08(\mathrm{td}, J=7.5$, $1.3 \mathrm{~Hz}, 2 \mathrm{H}), 7.02$ (dd, $J=7.9,1.4 \mathrm{~Hz}, 2 \mathrm{H}) .{ }^{13} \mathrm{C} \mathrm{NMR}\left(125 \mathrm{MHz}, \mathrm{CDCl}_{3}\right)$ : $\delta 195.38,147.75,142.16,138.00,132.65,132.36,132.10,129.80,129.11,128.25$, $128.18,127.16,125.00,122.87,120.60$. MS (MALDI-TOF): $\mathrm{m} / \mathrm{z}[\mathrm{M}]^{+}$calcd 379.10; found, 378.80. $\mathrm{C}_{25} \mathrm{H}_{17} \mathrm{NOS}$ : C, 79.13; $\mathrm{H}, 4.52 ; \mathrm{N}, 3.69$; found: $\mathrm{C}, 79.09$; $\mathrm{H}, 4.55 ; \mathrm{N}, 3.75$.

\section{Quantum chemical calculations}

All quantum chemical calculations were performed using the Gaussian 09 program package. ${ }^{40}$ Gas-phase geometry optimizations for the lowest-excited singlet and triplet states were carried out using time-dependent density functional theory calculations at the PBE0/6-31G(d) level. ${ }^{41}$

\section{Photophysical measurements}

UV-vis absorption and photoluminescence (PL) spectra were measured using a V-670Y spectrometer (Jasco, Tokyo, Japan) and an FP-8600Y
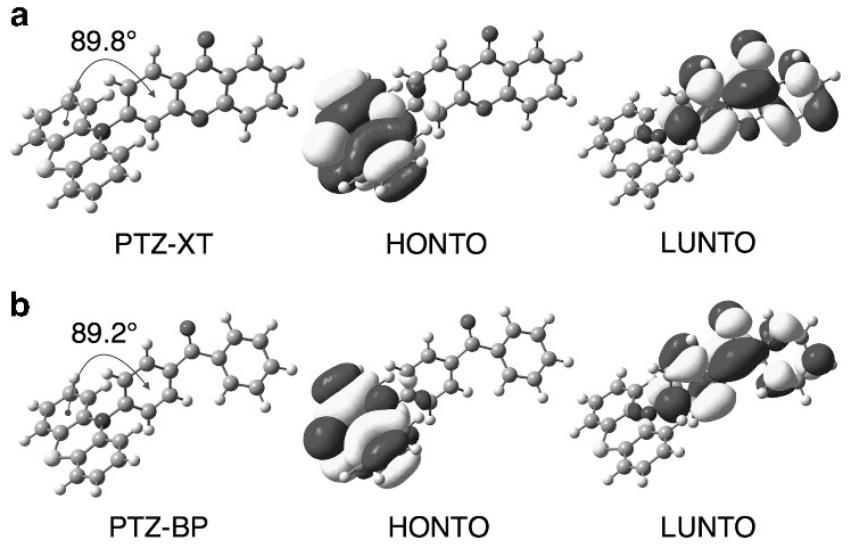

Figure 2 Configurations and the highest occupied (HO) and lowest unoccupied (LU) natural transition orbitals (NTOs) of the lowest-excited singlet states of (a) PTZ-XT and (b) PTZ-BP. The isovalue is 0.02 . A full color version of this figure is available at Polymer Journal online.

Table 1 Experimental photophysical properties of neat films of PTZ-XT and PTZ-BP

\begin{tabular}{lccccccc}
\hline & $\begin{array}{c}\lambda_{P L^{a}} \\
\text { Emitter }\end{array}$ & $\begin{array}{c}\Delta E_{S T}{ }^{b} \\
(m e V)\end{array}$ & $\begin{array}{c}\tau_{P F}{ }^{c} \\
(n s)\end{array}$ & $\begin{array}{c}\tau_{D F}{ }^{d} \\
(\mu s)\end{array}$ & $\begin{array}{c}\Phi_{P L^{e}} \\
(\%)\end{array}$ & $I_{P}{ }^{f}(\mathrm{eV})$ & $E_{A}{ }^{g}(\mathrm{eV})$ \\
\hline PTZ-XT & 545 & 71 & 29 & 1.9 & 53 & -5.5 & -2.6 \\
PTZ-BP & 565 & 65 & 23 & 1.4 & 31 & -5.6 & -2.6 \\
\hline
\end{tabular}

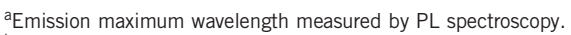

bSinglet-triplet energy splitting determined from the onsets of the fluorescence $(300 \mathrm{~K})$ and phosphorescence $(5 \mathrm{~K})$ spectra (see Supplementary Figure S1).

cTransient lifetime for prompt fluorescence measured at $300 \mathrm{~K}$ under $\mathrm{N}_{2}$.

dTransient lifetime for delayed fluorescence measured at $300 \mathrm{~K}$ under $\mathrm{N}_{2}$.

eAbsolute PL quantum yield measured at $300 \mathrm{~K}$ under $\mathrm{N}_{2}$.

${ }_{f}$ Ionization potential measured by photoelectron yield spectroscopy.

gElectron affinity determined from $/ p$ and optical bandgap determined from absorption onset.

spectrophotometer (Jasco), respectively. Absolute PL quantum yields were measured using an ILF-835 integrating sphere system (Jasco). Transient PL decay measurements were performed using a C11367 Quantaurus-Tau fluorescence lifetime spectrometer (Hamamatsu Photonics, Shizuoka, Japan).

\section{OLED fabrication and characterization}

ITO-coated glass substrates were cleaned with detergent, deionized water, acetone and isopropanol. The substrates were then subjected to a UV-ozone treatment for $15 \mathrm{~min}$, before they were loaded into a vacuum evaporation system. The organic layers were thermally evaporated on the substrates under vacuum $\left(<3 \times 10^{-4} \mathrm{~Pa}\right)$ with an evaporation rate of $<0.3 \mathrm{~nm} \mathrm{~s}^{-1}$. $\mathrm{Al}$ cathodes were then deposited through a shadow mask. Layer thicknesses and deposition rates were monitored in situ during deposition by an oscillating quartz thickness monitor. OLEDs were characterized using a Keithley 2400 source meter and a CS-2000 spectroradiometer (Konica Minolta, Tokyo, Japan).

\section{RESULTS AND DISCUSSION}

The key feature for producing efficient AIDF emitters is the use of an electron donor-acceptor system that adopts a twisted conformation to spatially separate the highest occupied molecular orbital and the lowest unoccupied molecular orbital. This molecular design reduces the electron exchange energy and hence leads to a small singlet-triplet energy splitting $\left(\Delta E_{\mathrm{ST}}\right)$, which can accelerate reverse intersystem crossing processes. Based on this design principle, we performed time-dependent density functional theory calculations for PTZ-XT and PTZ-BP (Figure 2). The dihedral angles between the donor 
and acceptor units in the lowest-excited singlet $\left(\mathrm{S}_{1}\right)$ states are approximately $90^{\circ}$ for both molecules, which gives rise to effective electronic decoupling between these units. As a result, the $\Delta E_{\mathrm{ST}}$ values of PTZ-XT and PTZ-BP were calculated to be 24 and $15 \mathrm{meV}$, respectively (see Table 1 and Supplementary Figure S1 for the
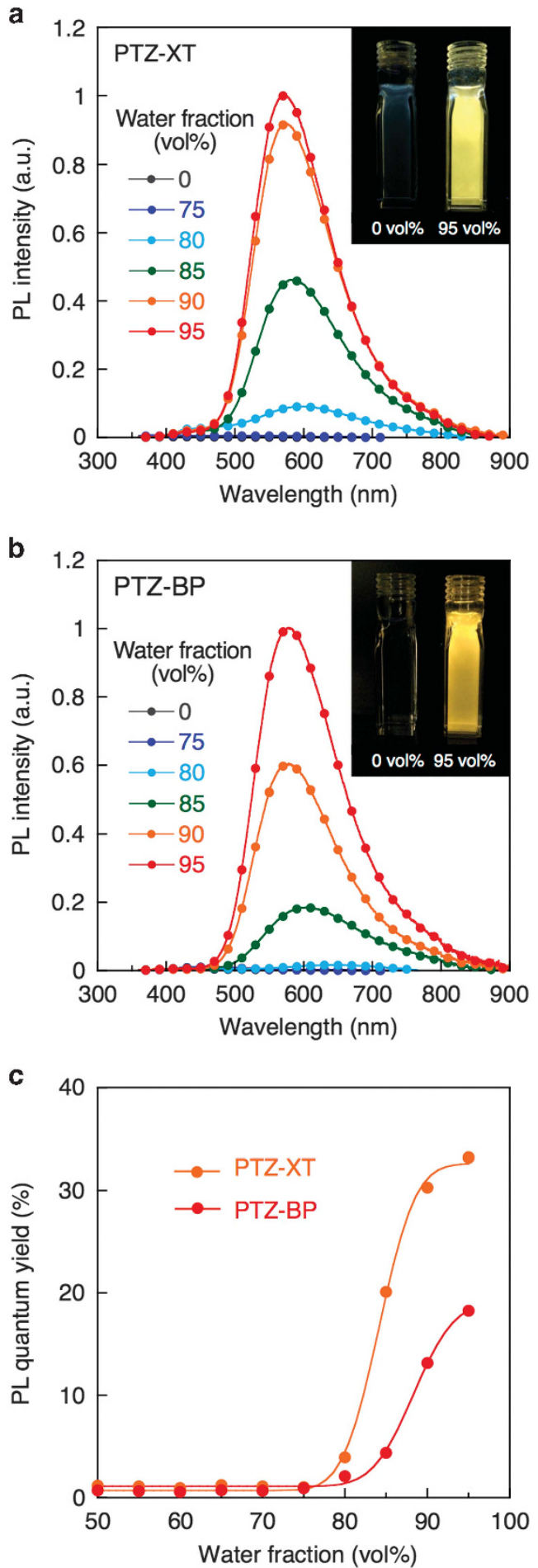

Figure $3 \mathrm{PL}$ spectra of (a) PTZ-XT and (b) PTZ-BP in the THF/water mixtures; the insets show photographs of $\mathrm{PL}$ emissions from $\mathrm{PTZ}-\mathrm{XT}$ and PTZ-BP in the THF/water mixtures under UV irradiation. (c) $\Phi_{\mathrm{PL}}$ of PTZ-XT and PTZ-BP as a function of the water fraction $\left(f_{\mathrm{w}}\right)$. experimental values). PTZ-XT and PTZ-BP were synthesized using the Buchwald-Hartwig reaction between $10 \mathrm{H}$-phenothiazine and 3-bromoxanthone or 4-bromobenzophenone. The molecular structures were identified by ${ }^{1} \mathrm{H}$ and ${ }^{13} \mathrm{C}$ NMR spectroscopy, MALDI-TOF mass spectrometry and elemental analysis (see the Experimental Section and Supplementary Figure S2-5 for details).

To examine whether PTZ-XT and PTZ-BP are AIE-active, we performed PL spectroscopic analysis on both compounds in the $\mathrm{THF} /$ water mixtures with water fractions $\left(f_{\mathrm{w}}\right)$ ranging from 0 to $95 \mathrm{vol}$ $\%$ (Figure 3). All the combinations of THF and water are miscible and the resulting mixtures form a single liquid phase at room temperature. As shown in Figure 3a, PTZ-XT exhibited a very weak blue emission with a maximum $\left(\lambda_{\mathrm{PL}}\right)$ of $480 \mathrm{~nm}$ at a $f_{\mathrm{w}}$ value below $75 \mathrm{vol} \%$. However, the net PL intensities increased abruptly upon the formation of colloidal aggregates when $f_{\mathrm{w}}$ increased above $80 \mathrm{vol} \%$. Meanwhile,

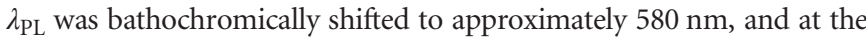
same time, the emission color changed to yellow. A large Stokes shift of $10700 \mathrm{~cm}^{-1}$ and a broad spectral shape of the emission suggest that the intense bright PL originates from the intramolecular charge-transfer excited state between the donor and acceptor units. At a $f_{\mathrm{w}}$ value of $95 \mathrm{vol} \%$, the PL quantum yield $\left(\Phi_{\mathrm{PL}}\right)$ of PTZ-XT reached 33\%, which is equivalent to an enhancement by a factor of 30 over the quantum yield of the THF solution $\left(f_{\mathrm{w}}=0 \mathrm{vol} \%\right)$ (Figure $3 \mathrm{c}$ ). A similar AIE behavior was also observed for PTZ-BP in THF/water mixtures (Figure 3b), whereas the only structural difference between the two compounds is that PTZ-XT contains an oxygen bridge, which
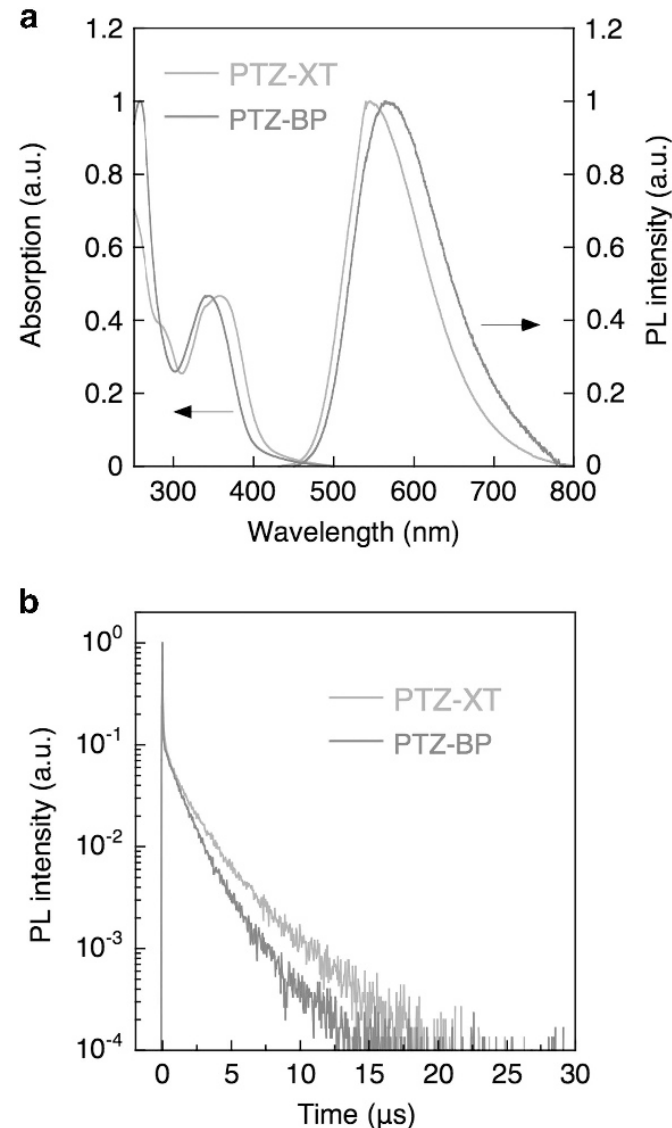

Figure 4 (a) UV - vis absorption and PL spectra of PTZ-XT and PTZ-BP neat films. (b) Transient PL decay curves of PTZ-XT and PTZ-BP neat films at $300 \mathrm{~K}$. A full color version of this figure is available at Polymer Journal online. 
restricts the internal rotations in the acceptor unit. Hence, the observed AIE behaviors of PTZ-XT and PTZ-BP are primarily attributed to aggregation-induced restriction of conformational changes in the phenothiazine donor unit ${ }^{42-44}$ and the rotation around the single bond between the donor and acceptor units.

In addition to the AIE behaviors of the aggregates in the THF/water mixtures, the two compounds display intense fluorescence in vacuum-deposited neat films. Neat films of PTZ-XT and PTZ-BP showed broad and structureless PL spectra with $\lambda_{\mathrm{PL}}$ at 545 and $565 \mathrm{~nm}$, respectively (Table 1 and Figure $4 \mathrm{a}$ ). The $\Phi_{\mathrm{PL}}$ values of the PTZ-XT and PTZ-BP neat films reached 53 and 31\%, respectively, demonstrating that these highly luminescent materials are viable candidates for use in non-doped emission layers for OLEDs. Furthermore, time-resolved transient PL analysis revealed doubleexponential decays for both compounds (Figure 4b), which are composed of prompt fluorescence with transient lifetimes $\left(\tau_{\mathrm{PF}}\right)$ of several tens of nanoseconds and delayed fluorescence with lifetimes $\left(\tau_{\mathrm{DF}}\right)$ of several microseconds (Table 1 and Supplementary Table S1). The delayed fluorescence was spectrally identical to the corresponding prompt fluorescence, and it supported the efficient up-conversion of non-radiative triplet excitons to radiative singlet excitons. In comparison, THF solutions of the PTZ-XT and PTZ-BP exhibited single-exponential decay profiles with only prompt fluorescence, which confirms that these materials are AIDF emitters.

To demonstrate the benefits of the AIDF emitters for optoelectronic applications, we fabricated OLEDs by employing non-doped emission layers. Although complex co-evaporation processes are required for common doped emission layers, the high values of the solid-state $\Phi_{\mathrm{PL}}$ of the AIDF emitters allowed us to fabricate efficient non-doped emission layers without any host materials, thus simplifying both the device architecture and the fabrication process. The non-doped OLEDs are composed of the following layer sequence: indium tin oxide (ITO, $100 \mathrm{~nm}) / 4,4^{\prime}$-bis[ $N$-(1-naphthyl)- $N$-phenylamino]1,1'-biphenyl $(\alpha$-NPD, $40 \mathrm{~nm}) / 3,3^{\prime}$-bis(carbazol-9-yl)-1,1'-biphenyl (mCBP, $10 \mathrm{~nm}) / \mathrm{AIDF}$ emitter $(15 \mathrm{~nm}) / 3,5,3^{\prime \prime}, 5^{\prime \prime}$-tetra-3-pyridyl$\left[1,1^{\prime} ; 3^{\prime}, 1^{\prime}\right]$ terphenyl (B3PyPB, $\left.55 \mathrm{~nm}\right)^{45} / 8$-hydroxyquinolinato lithium (Liq, $1 \mathrm{~nm}) / \mathrm{Al}(80 \mathrm{~nm})$. A schematic energy-level diagram of the OLEDs is shown in Figure 5a, in which the non-doped emission layers containing the AIDF emitters are sandwiched between wide bandgap materials, $\mathrm{mCBP}$ and $\mathrm{B} 3 \mathrm{PyPB}$ (Figure 5b), to confine the electrogenerated excitons within the emission layers. The EL spectra, current density-voltage-luminance, and $\eta_{\text {ext }}$-current density characteristics of the fabricated OLEDs are shown in Figure $5 c$, d and e, respectively. Both devices using PTZ-XT and PTZ-BP as the non-doped emission layer exhibited EL spectra that are characteristic of the AIDF emitters, with emission maxima $\left(\lambda_{\mathrm{EL}}\right)$ at 553 and $577 \mathrm{~nm}$, respectively. No additional emission from the adjacent layers was observed in the devices, suggesting that the electrogenerated excitons were well confined within the emission layers. Maximum $\eta_{\text {ext }}$ values as high as 11.1 and $7.6 \%$ were obtained for the PTZ-XT- and PTZ-BP-based devices, respectively. By assuming a light outcoupling efficiency of $20 \%$, the corresponding $\eta_{\text {int }}$ values were estimated to be 56 and 38\%, which are much higher than the theoretical maximum $\eta_{\text {int }}$ value of $25 \%$ for conventional fluorescent OLEDs. These high EL efficiencies therefore demonstrate that the electrogenerated triplet excitons are effectively up-converted to the radiative singlet excitons in the AIDF emitters, which results in the enhanced $\eta_{\text {int }}$. a

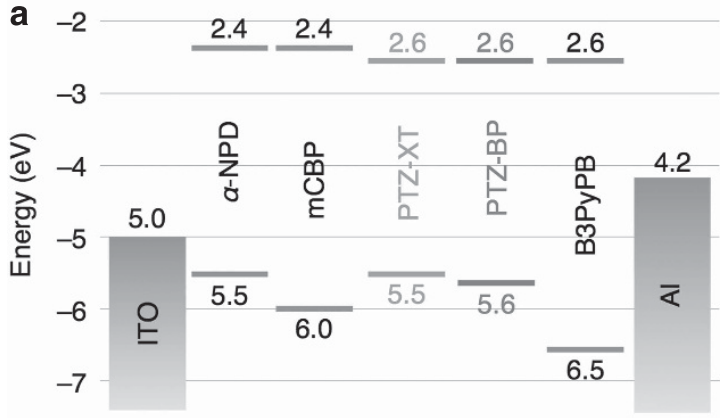

b
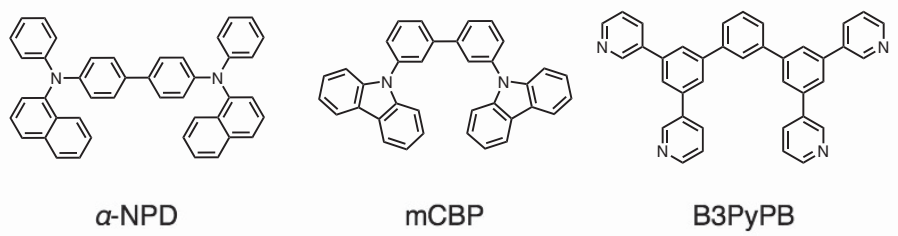

C

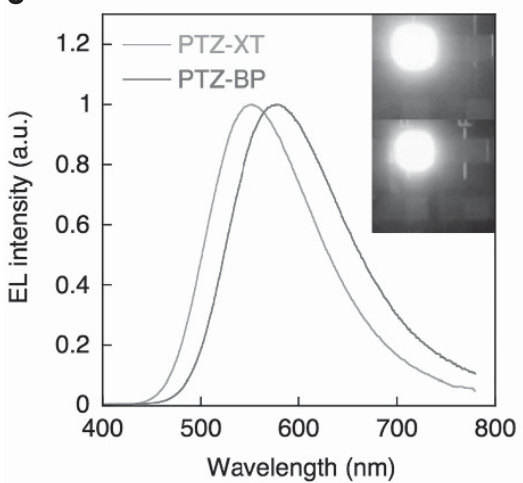

d

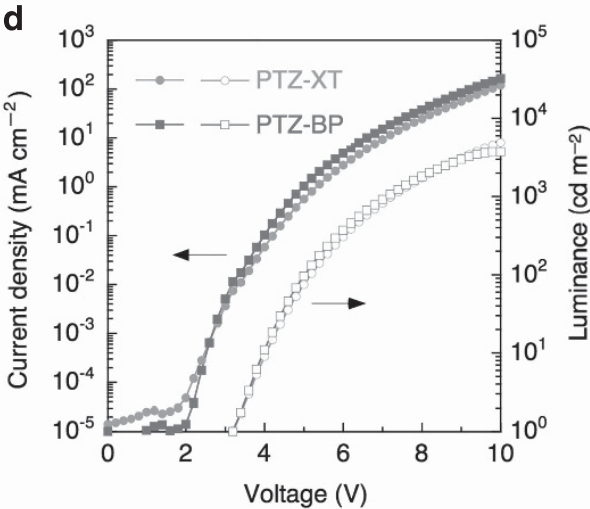

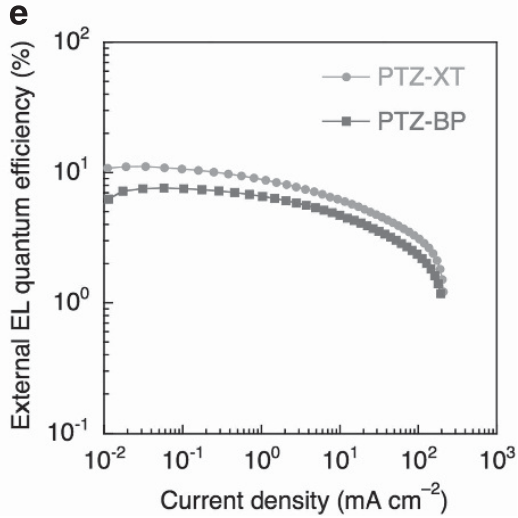

Figure 5 (a) Schematic energy-level diagram of non-doped OLEDs based on PTZ-XT and PTZ-BP as AIDF emitters. (b) Molecular structures of the materials used. (c) EL spectra of the non-doped OLEDs operating at $10 \mathrm{~mA} \mathrm{~cm}^{-2}$; the insets show the photographs of the devices based on (top) PTZ-XT and (bottom) PTZ-BP. (d) Current density-voltage-luminance characteristics of the non-doped OLEDs. (e) $\eta_{\text {ext }}$-current density characteristics of the non-doped OLEDs. A full color version of this figure is available at Polymer Journal online. 


\section{CONCLUSIONS}

We have successfully developed and characterized two donor-acceptor luminescent molecules, PTZ-XT and PTZ-BP. Both materials were found to exhibit efficient delayed fluorescence upon aggregation. By taking advantage of their unique AIDF properties, we achieved high $\eta_{\text {ext }}$ values of up to $11.1 \%$ by using these materials as non-doped emission layers in OLEDs. These results demonstrate that the AIDF emitters are viable candidates for use in highly simplified non-doped OLEDs.

\section{CONFLICT OF INTEREST}

The authors declare no conflict of interest.

\section{ACKNOWLEDGEMENTS}

This work was supported in part by KAKENHI (Grant No. 15H01049, 25708032, 26620168 and 15H06470) from the Japan Society for the Promotion of Science (JSPS), and by the Canon Foundation, the Kurata Memorial Hitachi Science and Technology Foundation, the Sumitomo Electric Group CSR Foundation, the Futaba Electronics Memorial Foundation and the Foundation for the Promotion of Ion Engineering.

1 Tang, C. W. \& VanSlyke, S. A. Organic electroluminescent diodes. Appl. Phys. Lett. 51, 913-915 (1987)

2 Mattoussi, H., Murata, H., Merritt, C. D., Iizumi, Y., Kido, J. \& Kafafi, Z. H. Photoluminescence quantum yield of pure and molecularly doped organic solid films. J. Appl. Phys. 86, 2642-2650 (1999)

3 Baldo, M. A., Adachi, C. \& Forrest, S. R. Transient analysis of organic electrophosphorescence. II. Transient analysis of triplet-triplet annihilation. Phys. Rev. B 62 10967-10977 (2000).

4 Swanson, S. A., Wallraff, G. M., Chen, J. P., Zhang, W., Bozano, L. D., Carter, K. R., Salem, J. R., Villa, R. \& Scott, J. C. Stable and efficient fluorescent red and green dyes for eternal and internal conversion of blue OLED emission. Chem. Mater. 15 2305-2312 (2003).

5 Kawamura, Y., Goushi, K., Brooks, J., Brown, J. J., Sadabe, H. \& Adachi, C. Intermolecular interaction and a concentration-quenching mechanism of phosphorescent Ir(III) complexes in a solid film. Phys. Rev. Lett. 96, 017404 (2006).

6 Luo, J., Xie, Z., Lam, J. W., Cheng, L., Chen, H., Quu, C., Kwok, H. S., Zhan, X., Liu, Y., Zhu, D. \& Tang, B. Z. Aggregation-induced emission of 1-methyl-1,2,3,4,5-pentaphenylsilole. Chem. Commun. 37, 1740-1741 (2001).

7 Tang, B. Z., Zhan, X., Yu, G., Lee, P. P. S., Liu, Y. \& Zhu, D. Efficient blue emission from siloles. J. Mater. Chem. 11, 2974-2978 (2001).

8 Chen, J., Law, C. C., Lam, J. W., Dong, Y., Lo, S. M., Williams, I. D., Zhu, D. \& Tang, B. Z. Synthesis, light emission, nanoaggregation, and restricted intramolecular rotation of 1,1-substituted 2,3,4,5-tetraphenylsiloles. Chem. Mater. 15, 1535-1546 (2003).

9 Yu, G., Yin, S., Liu, Y., Chen, J., Xu, X., Sun, X., Ma, D., Zhan, X., Peng, Q. \& Shuai, Z. Structures, electronic states, photoluminescence, and carrier transport properties of 1,1-disubstituted 2,3,4,5-tetraphenylsiloles. J. Am. Chem. Soc. 127, 6335-6346 (2005).

10 An, B.-K., Kwon, S.-K., Jung, S.-D. \& Park, S. Y. Enhanced emission and its switching in fluorescent organic nanoparticles. J. Am. Chem. Soc. 124, 14410-14415 (2002).

11 An, B.-K., Lee, D.-S., Lee, J.-S., Park, Y.-S., Song, H.-S. \& Park, S. Y. Strongly fluorescent organogel system comprising fibrillar self-assembly of a trifluoromethylbased cyanostilbene derivative. J. Am. Chem. Soc. 126, 10232-10233 (2004).

12 An, B.-K., Gierschner, J. \& Park, S. Y. $\pi$-Conjugated cyanostilbene derivatives: a unique self-assembly motif for molecular nanostructures with enhanced emission and transport. Acc. Chem. Res. 45, 544-554 (2012).

13 Kokado, K. \& Chujo, Y. Emission via aggregation of alternating polymers with $o$-carborane and $p$-phenylene-ethynylene sequences. Macromolecules 42, 1418-1420 (2009).

14 Peterson, J. J., Werre, M., Simon, Y. C., Coughlin, E. B. \& Carter, K. R. Carboranecontaining polyfluorene: o-carborane in the main chain. Macromolecules 42, 8594-8598 (2009).

15 Kokado, K. \& Chujo, Y. Multicolor tuning of aggregation-induced emission through substituent variation of diphenyl-o-carborane. J. Org. Chem. 76, 316-319 (2010).

16 Tominaga, M., Naito, H., Morisaki, Y. \& Chujo, Y. Colour-tunable aggregation-induced emission of trifunctional o-carborane dyes. New J. Chem. 38, 5686-5690 (2014).

17 Kim, S.-Y., Cho, Y.-J., Jin, G. F., Han, W.-S., Son, H.-J., Cho, D. W. \& Kang, S. O. Intriguing emission properties of triphenylamine-carborane systems. Phys. Chem. Chem. Phys. 17, 15679-15682 (2015).

18 Huang, J., Yang, X., Li, X., Chen, P., Tang, R., Li, F., Lu, P., Ma, Y., Wang, L. \& Qin, J. Bipolar AlE-active luminogens comprised of an oxadiazole core and terminal TPE moieties as a new type of host for doped electroluminescence. Chem. Commun. 48, 9586-9588 (2012).
19 Li, H., Chi, Z., Zhang, X., Xu, B., Liu, S., Zhang, Y. \& Xu, J. New thermally stable aggregation-induced emission enhancement compounds for non-doped red organic light-emitting diodes. Chem. Commun. 47, 11273-11275 (2011).

20 Liu, Y., Chen, S., Lam, J. W., Lu, P., Kwok, R. T., Mahtab, F., Kwok, H. S. \& Tang, B. Z. Tuning the electronic nature of aggregation-induced emission luminogens with enhanced hole-transporting property. Chem. Mater. 23, 2536-2544 (2011).

21 Zhao, Z., Lam, J. W. Y. \& Tang, B. Z. Tetraphenylethene: a versatile AIE building block for the construction of efficient luminescent materials for organic light-emitting diodes. J. Mater. Chem. 22, 23726-23740 (2012).

22 Kim, J. Y., Yasuda, T., Yang, Y. S. \& Adachi, C. Bifunctional star-burst amorphous molecular materials for OLEDs: achieving highly efficient solid-state luminescence and carrier transport induced by spontaneous molecular orientation. Adv. Mater. 25, 2666-2671 (2013).

23 Mei, J., Hong, Y., Lam, J. W., Qin, A., Tang, Y. \& Tang, B. Z. Creation of bifunctional materials: improve electron-transporting ability of light emitters based on AIE-active 2,3,4,5-tetraphenylsiloles. Adv. Mater. 26, 5429-5479 (2014).

24 Segal, M., Baldo, M., Holmes, R., Forrest, S. \& Soos, Z. Excitonic singlet-triplet ratios in molecular and polymeric organic materials. Phys. Rev. B 68, 075211 (2003).

25 Furue, R., Nishimoto, T., Park, I. S., Lee, J. \& Yasuda, T. Aggregation-induced delayed fluorescence based on donor/acceptor-tethered Janus carborane triads: unique photophysical properties of nondoped OLEDs. Angew. Chem. Int. Ed. 55, 7171-7175 (2016).

26 Li, J., Jiang, Y., Cheng, J., Zhang, Y., Su, H., Lam, J. W., Sung, H. H., Wong, K. S. Kwok, H. S. \& Tang, B. Z. Tuning the singlet-triplet energy gap of AIE luminogens: crystallization-induced room temperature phosphorescence and delay fluorescence, tunable temperature response, highly efficient non-doped organic light-emitting diodes. Phys. Chem. Chem. Phys. 17, 1134-1141 (2015).

27 Xie, Z., Chen, C., Xu, S., Li, J., Zhang, Y., Liu, S., Xu, J. \& Chi, Z. White-light emission strategy of a single organic compound with aggregation-induced emission and delayed fluorescence properties. Angew. Chem. Int. Ed. 54, 7181-7184 (2015).

28 Xu, S., Liu, T., Mu, Y., Wang, Y. F., Chi, Z., Lo, C. C., Liu, S., Zhang, Y., Lien, A. \& Xu, J. An organic molecule with asymmetric structure exhibiting aggregation-induced emission, delayed fluorescence, and mechanoluminescence. Angew. Chem. Int. Ed. 54, 874-878 (2015).

29 Gan, S., Luo, W., He, B., Chen, L., Nie, H., Hu, R., Qin, A., Zhao, Z. \& Tang, B. Z Integration of aggregation-induced emission and delayed fluorescence into electronic donor-acceptor conjugates. J. Mater. Chem. C 4, 3705-3708 (2016).

30 Sun, K., Jiang, W., Ban, X., Huang, B., Zhang, Z., Ye, M. \& Sun, Y. Novel aggregationinduced emission and thermally activated delayed fluorescence materials based on thianthrene- $9,9^{\prime}, 10,10^{\prime}$-tetraoxide derivatives. RSC Adv. 6 , 22137-22143 (2016).

31 Endo, A., Sato, K., Yoshimura, K., Kai, T., Kawada, A., Miyazaki, H. \& Adachi, C. Efficient up-conversion of triplet excitons into a singlet state and its application for organic light emitting diodes. Appl. Phys. Lett. 98, 083302 (2011).

32 Uoyama, H., Goushi, K., Shizu, K., Nomura, H. \& Adachi, C. Highly efficient organic light-emitting diodes from delayed fluorescence. Nature 492 234-238 (2012).

33 Dias, F. B., Bourdakos, K. N., Jankus, V., Moss, K. C., Kamtekar, K. T., Bhalla, V., Santos, J., Bryce, M. R. \& Monkman, A. P. Triplet harvesting with $100 \%$ efficiency by way of thermally activated delayed fluorescence in charge transfer OLED emitters. Adv. Mater. 25, 3707-3714 (2013).

34 Zhang, Q., Li, B., Huang, S., Nomura, H., Tanaka, H. \& Adachi, C. Efficient blue organic light-emitting diodes employing thermally activated delayed fluorescence. Nat. Photon 8, 326-332 (2014).

35 Lee, S. Y., Yasuda, T., Yang, Y. S., Zhang, Q. \& Adachi, C. Luminous butterflies: efficient exciton harvesting by benzophenone derivatives for full-color delayed fluorescence OLEDs. Angew. Chem. Int. Ed. 53, 6402-6406 (2014).

36 Hirata, S., Sakai, Y., Masui, K., Tanaka, H., Lee, S. Y., Nomura, H., Nakamura, N., Yasumatsu, M., Nakanotani, H. \& Zhang, Q. Highly efficient blue electroluminescence based on thermally activated delayed fluorescence. Nat. Mater. 14, 330-336 (2015).

37 Zhang, Q., Tsang, D., Kuwabara, H., Hatae, Y., Li, B., Takahashi, T., Lee, S. Y., Yasuda, T. \& Adachi, C. Nearly $100 \%$ internal quantum efficiency in undoped electroluminescent devices employing pure organic emitters. Adv. Mater. 27, 2096-2100 (2015).

38 Numata, M., Yasuda, T. \& Adachi, C. High efficiency pure blue thermally activated delayed fluorescence molecules having $10 \mathrm{H}$-phenoxaborin and acridan units. Chem. Commun. 51, 9443-9446 (2015).

39 Park, I. S., Lee, S. Y., Adachi, C. \& Yasuda, T. Full-color delayed fluorescence materials based on wedge-shaped phthalonitriles and dicyanopyrazines: systematic design, tunable photophysical properties, and OLED performance. Adv. Funct. Mater. 26, 1813-1821 (2016).

40 Frisch, M. J., Trucks, G. W., Schlegel, H. B., Scuseria, G. E., Robb, M. A., Cheeseman, J. R., Scalmani, G., Barone, V., Mennucci, B., Petersson, G. A., Nakatsuji, H., Caricato, M., Li, X., Hratchian, H. P., Izmaylov, A. F., Bloino, J., Zheng, G., Sonnenberg, J. L., Hada, M., Ehara, M., Toyota, K., Fukuda, R., Hasegawa, J., Ishida, M., Nakajima, T., Honda, Y., Kitao, O., Nakai, H., Vreven, T., Montgomery, J. A. Jr., Peralta, J. E., Ogliaro, F., Bearpark, M., Heyd, J. J., Brothers, E., Kudin, K. N., Staroverov, V. N., Kobayashi, R., Normand, J., Raghavachari, K., Rendell, A., Burant, J. C., Iyengar, S. S., Tomasi, J., Cossi, M., Rega, N., Millam, J. M., Klene, M., Knox, J. E., Cross, J. B., Bakken, V., Adamo, C., Jaramillo, J., Gomperts, R., Stratmann, R. E., Yazyev, O., Austin, A. J., Cammi, R., 
Pomelli, C., Ochterski, J. W., Martin, R. L., Morokuma, K., Zakrzewski, V. G., Voth, G. A., Salvador, P., Dannenberg, J. J., Dapprich, S., Daniels, A. D., Farkas, Ö., Foresman, J. B., Ortiz, J. V., Cioslowski, J. \& Fox, D. J. Gaussian 09, Revision E.O1 (Gaussian, Inc., Wallingford CT, 2009).

41 Adamo, C. \& Barone, V. Toward reliable density functional methods without adjustable parameters: The PBEO model. J. Chem. Phys. 110, 6158-6170 (1999).

42 Daub, J., Engl, R., Kurzawa, J., Miller, S., Schneider, S., Stockmann, A. \& Wasielewski, M. R. Competition between conformational relaxation and intramolecular electron transfer within phenothiazine-pyrene dyads. J. Phys. Chem. A 105 , 5655-5665 (2001).
43 Stockmann, A., Kurzawa, J., Fritz, N., Acar, N., Schneider, S., Daub, J., Engl, R. \& Clark, T. Conformational control of photoinduced charge separation within phenothiazine-pyrene dyads. J. Phys. Chem. A 106, 7958-7970 (2002).

44 Tanaka, H., Shizu, K., Nakanotani, H. \& Adachi, C. Dual intramolecular charge-transfer fluorescence derived from a phenothiazine-triphenyltriazine derivative. J. Phys. Chem. $C$ 118, 15985-15994 (2014).

45 Sasabe, H., Gonmori, E., Chiba, T., Li, Y.-J., Tanaka, D., Su, S.-J., Takeda, T., Pu, Y.-J., Nakayama, K.-I. \& Kido, J. Wide-energy-gap electron-transport materials containing 3,5-dipyridylphenyl moieties for an ultra high efficiency blue organic light-emitting device. Chem. Mater. 20, 5951-5953 (2008).

Supplementary Information accompanies the paper on Polymer Journal website (http://www.nature.com/pj) 